\title{
Scheduling optimization of mixed model assembly lines Based on Genetic Algorithms
}

\author{
Shuo Cheng ${ }^{1,2, a}$, Minghai Yuan ${ }^{1,2, b}$, Shuyang $\operatorname{Yan}^{1,2, c}$ \\ ${ }^{1}$ College of Mechanical and Electrical Engineering, Hohai university ,Jiangsu Changzhou,China; \\ ${ }^{2}$ Huaian Research Institute of Hohai University , Jiangsu Huaian,China; \\ a710609726@qq.com, bymhai@126.com, c1737364825@qq.com
}

Keywords: Mixed model assembly lines; Scheduling; Objective function; Genetic Algorithms

\begin{abstract}
For meeting diversified demand of customers without the need for large product inventories an objective function on maintaining the uniform of a spare parts consumption rate was put forward on the basis of summarizing the scheduling problem with mixed flow assembly line, and the mathematical model of the objective function was established. then a kind of genetic algorithm was presented to solve the mixed flow assembly line scheduling model. Finally, a simulation example was employed to illustrate the validity and effectiveness of the proposed method.
\end{abstract}

\section{Introduction}

With the increasingly fierce market competition, every enterprise is looking for a better solution, in order to improve the efficiency of enterprise production, operation and management, so as to improve the core competitive advantage of enterprises. And the core of production and operations management is whether the production scheduling problem could obtain the optimal solution efficiently. In view of this situation, mixed flow assembly line scheduling is an assorted optimization problem we need to optimize [1, 2, 3]. This paper adopts a kind of genetic algorithm to optimize the workshop scheduling, it can make the production maintain a state of equilibrium and continuous flow to improve the utilization rate of personnel and equipment, by mean of the sorting of mixed flow assembly line, different product assembly sequence can be decided to improve the utilization rate of the mixed flow assembly line, finally, the enterprise production efficiency can be improved and the production costs can be reduced.

\section{Scheduling optimization of mixed model assembly lines}

Scheduling optimization of mixed model assembly lines is aimed to solve the production load equilibrium problems between the assembly line and the entire production system, to to avoid the system bottlenecks[4]. As shown in figure 1, the assembly line is assembling two type B car, one type $\mathrm{C}$ car, and a type A car is about to enter the assembly line, Sub assembly unit will have different demands for parts according to the difference of vehicle models which enter the assembly line, however, In JIT(Just in Time) production environment, if each production unit maintains lower WIP (Work In Process) inventory, it will cause the phenomenon of imbalance between supply and demand. therefore, the scheduling of total assembly line has the important influence on the production of the whole system of load balancing and the implementation of JIT target[5]. 


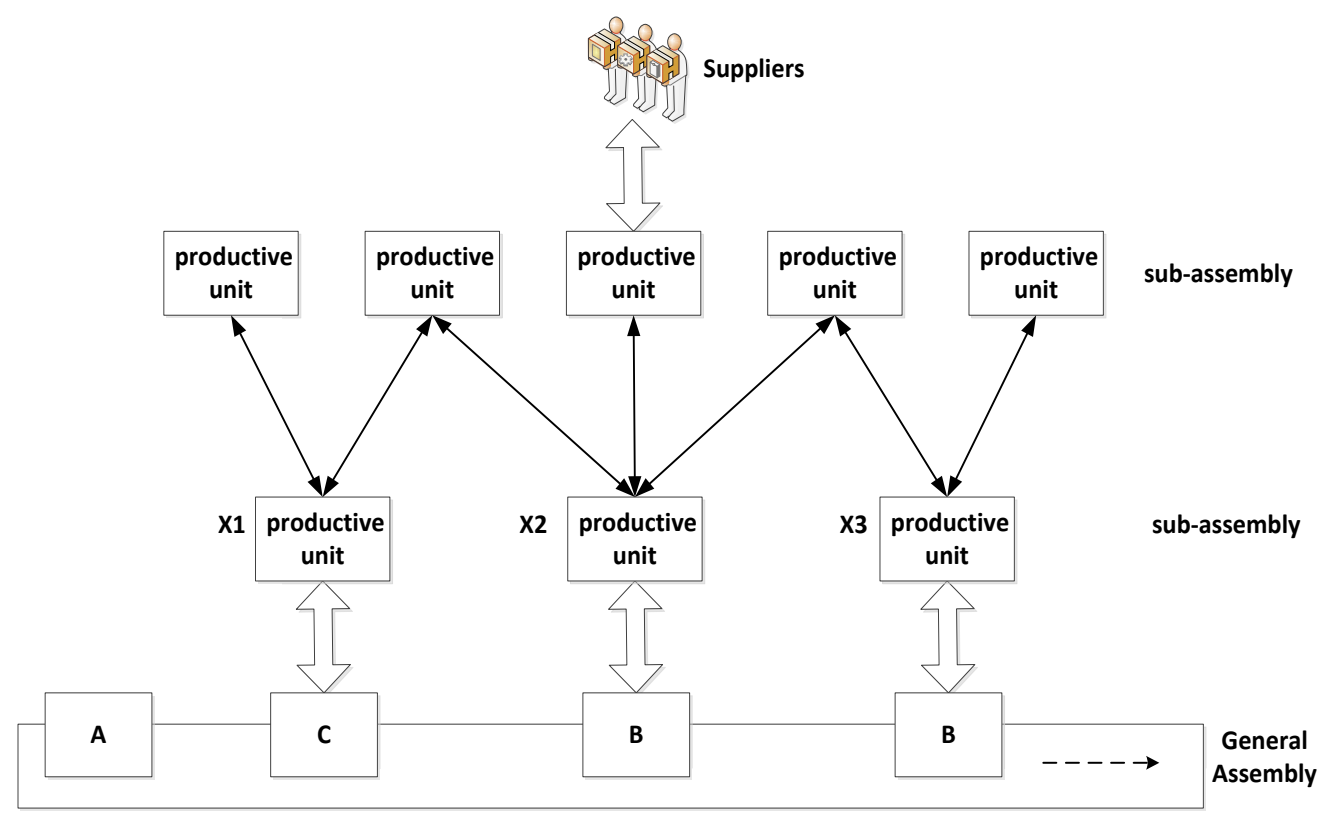

Fig.1 Total assembly line of JIT

\section{Model of mixed model assembly lines scheduling}

For batch manufacturing products, cycle sequencing method is used in practical production, for example, on a mixed assembly line of a manufacturing automobile enterprise, the demand of production m models are respectively $D_{1}, D_{2}, D_{3}, \cdots, D_{n}$, and the greatest common divisor is $d$, setting $d_{i}=D_{i} / d(i=1,2, \cdots, n)$, in the production operation , we just need sort the vehicle model which set $D=\sum_{i=1}^{n} d_{i}$ as the unit, this is called a cyclic process. Repeated $d$ cyclic processes, An order for all products during a plan process will be achieved. this paper is setting a production order for the circulation process.

Because the number of type of car on the mixed model assembly lines is $n$, in a production cycle, the demand for $n$ models is respectively $d_{1}, d_{2}, \cdots, d_{n}$, then in the production cycle during a plan, the total demand of the $n$ kinds of car is $D=\sum_{i=1}^{n} d_{i}$. Setting $b_{i j}$ as the number of parts $j(j=1,2, \cdots, m)$ for the assembly model $i(i=1,2, \cdots, n)$, for it, the total demand $T_{j}$ of parts $j$ in a productive cycle can be figured out, and the total demand is as: $T_{j}=\sum_{i=1}^{n} b_{i j} \cdot d_{i}$.

During a circle in the plan, the total demand of $n$ kinds of car is $D$, so the ideal demand $\alpha_{j}$ is as

$$
\alpha_{j}=\frac{\sum_{i=1}^{n} b_{i j} d_{i}}{D} \text {. and the ideal demand of former } k \text { is } k \cdot \alpha_{j} \text {. And if the kind of the } k \text { location is } i
$$

$(i=1,2, \cdots, n)$,the $X_{k i}$ will be 1 ,so it can be exported that the actual number of consumption parts of the former $k$ cars in the production scheduling is: $\beta_{k j}=\beta_{k-1, j}+X_{k i} \cdot b_{i j}$. Therefore, after a series of derivation, within a production cycle, the final scheduling objective function for production on mixed model assembly lines is as followed :

$$
F=\min \sum_{k=1}^{D} \sum_{i=1}^{n} \sum_{j=1}^{m}\left(k \cdot \alpha_{j}-b_{i j}-\beta_{k-1, j}\right)^{2} X_{k i}
$$




\section{Genetic algorithm for mixed model assembly lines scheduling}

Gene encoding. If the model number is treated as the encoding of genetic algorithm, a genetic string will represent one kind of production scheduling, and its genetic value on each locus represents the product number , the length of the gene string is equal to the total input of n models in a minimum production cycle.

The generation of initial population. The generation of initial population adopts completely random method. According to the requirement of the production assembly, the amount and type of assembly model which meet the minimum production cycle can be determined, on this basis, we can generate the gene string at random.

The design of fitness function. In this paper, the objective function is the fitness function at this time, it just need a little change in the selection process.

Genetic operations. In this paper, we adopt the method of elite selection to select the operator. Firstly, we just need figure out the fitness value of each individual ,and the fitness value should be sorted from small to large.Then this article adopts the way of partial matching and cross restructuring to cross operator.In order to increase the number of mutated efficient solution, when the mutation operator proceeds, we just need randomly select two loci in the gene string and exchange the genic value of this two loci, like this, an individual mutation operation is completed. To sum up, the taken by this article is shown in figure 2 .

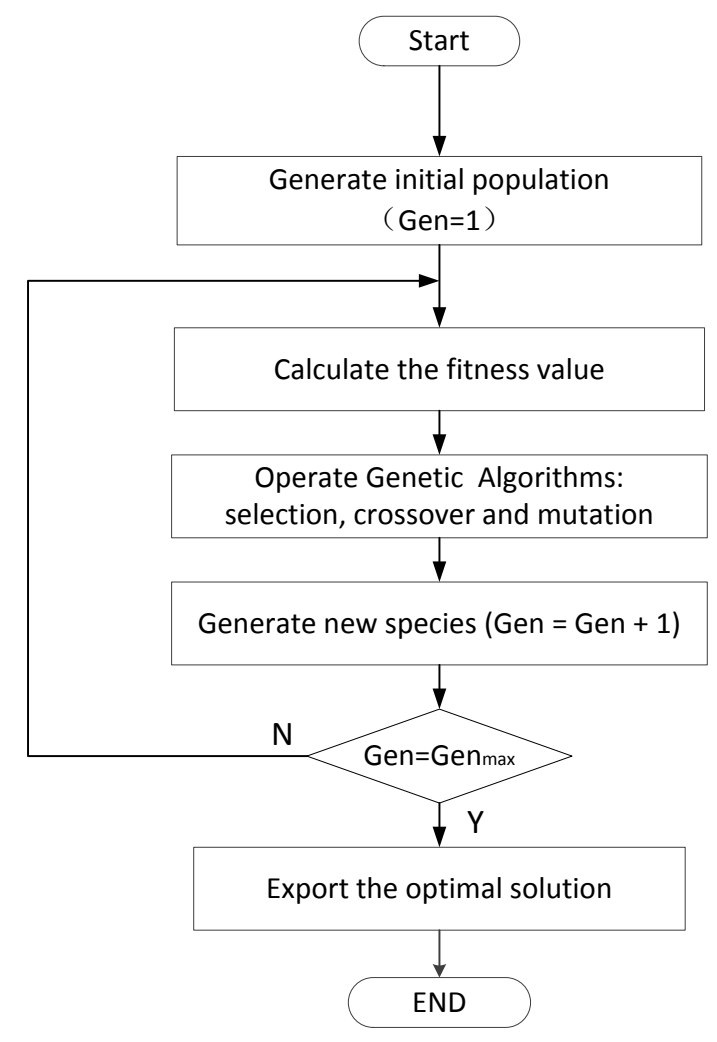

Fig. 2 Flow chart of genetic algorithm

\section{Simulation example}

To verify the validity of the genetic algorithm program, this paper adopts the data of literature [4] .The experimental data are shown in Table 1 . 
Table 1 The number of each model and parts consumption

\begin{tabular}{|c|c|c|c|c|c|c|c|c|c|c|c|}
\hline & \multirow{2}{*}{$\begin{array}{c}\text { Number of } \\
\text { Vehicle } \\
\text { model } \\
\text { vehicle model in a } \\
\text { productive cycle }\end{array}$} & \multicolumn{7}{|c|}{ Number of parts required } \\
\cline { 3 - 14 } & & 2 & 3 & 4 & 5 & 6 & 7 & 8 & 9 & 10 \\
\hline 1 & 5 & 0 & 17 & 9 & 0 & 4 & 0 & 0 & 18 & 0 & 0 \\
\hline 2 & 2 & 12 & 13 & 0 & 11 & 0 & 0 & 0 & 1 & 17 & 17 \\
\hline 3 & 3 & 2 & 4 & 0 & 19 & 0 & 12 & 6 & 4 & 9 & 3 \\
\hline 4 & 3 & 0 & 15 & 0 & 19 & 0 & 15 & 9 & 9 & 0 & 6 \\
\hline 5 & 3 & 0 & 0 & 5 & 7 & 8 & 10 & 4 & 0 & 0 & 0 \\
\hline $\begin{array}{c}\text { Total number of parts in a } \\
\text { productive cycle }\end{array}$ & 30 & 168 & 60 & 157 & 44 & 111 & 57 & 131 & 61 & 61 \\
\hline
\end{tabular}

The result using genetic algorithm to get the sorting is as shown in Table 2.

Table 2 Result with genetic algorithm

\begin{tabular}{|c|c|c|}
\hline Algorithm & Sorting result & $\begin{array}{c}\text { The objective function } \\
\text { value }\end{array}$ \\
\hline $\begin{array}{c}\text { The algorithm in this } \\
\text { paper }\end{array}$ & $\begin{array}{c}3-1-4-2-5-1-4-5-1-3-1-2- \\
5-4-1-3\end{array}$ & 3073.00 \\
\hline
\end{tabular}

\section{Summary}

For meeting diversified demand of customers without the need for large product inventories, this paper set up an objective function on maintaining the uniform of a spare parts consumption rate and adopted the genetic algorithm to solve the model as the Simulation example showed, the model and method is correct and effective.

\section{Acknowledgments}

This work was supported by Applied Basic Research Programs of Changzhou under grant number CJ20140027, This work was supported by the open foundation of Huaian Research Institute of Hohai University, and the postgraduate innovation project of Jiangsu province.

\section{References}

[1] G.H.Liu:The Study on Flexible Resoucres Job Shop Scheduling Based on Genetic Algorithm (2006)

[2] Zh.H.Liu: Study for Production Sequencing of Mixed-Model Assembly Lines in Just-In-Time Production Mode (2004)

[3] X.B. Zhao,Zh.Y. Zhou and Ainishet Asres,in:A note on Toyota's goal of sequencing mixed models on an assembly line,edtied by Computers\&Industrial Engineering(1999).

[4] W.Zhao,W.x. Han and Y.T. Luo,in Scheduling mixed-model assembly lines in JIT production systems. Journal of management science(2000) 
[5] W.J.Jin and W.H Ye:Study on the Barcode Based WIP Tracking and Management System under the Lean Production Mode,edtied by Machine Building \& Automation(2006) 